\title{
A two-stage retrospective analysis to determine the effect of entry point on higher exit of proximal pins in lateral pinning of supracondylar humerus fracture in children
}

\author{
Xianglu Ji, Allieu Kamara, Enbo Wang ${ }^{*}$ (D) Tianjing Liu, Liwei Shi and Lianyong Li
}

\begin{abstract}
Background: Kirschner wire fixation remains to be the mainstream treatment modality in unstable or displaced supracondylar humerus fracture in children, with divergent lateral pins being the most preferred due to their sufficient stability and decreased risk of ulnar nerve injury. However, the entry point at which the proximal lateral pin can be inserted to achieve a more proximal exit and maximum divergence has not been reported. This study retrospectively analyzed the characteristics and factors influencing the entry and exit points of the proximal lateral pins.

Methods: The study was divided into two stages. In stage one, the entry and exit points of the proximal pins of lateral pinning configuration were analyzed from intra-operative radiographs of children treated for extension-type supracondylar humerus fractures. The coronal and sagittal pin angles formed by the proximal pins were also measured. Using the findings of stage one, we intentionally tried to achieve a more proximal exit with the proximal pins in stage two. Comparisons between groups of patients treated by random and intentional pinnings were done statistically.

Results: In the first stage, 47 (29.2\%) of the 161 proximal pins exited above the metaphyseal-diaphyseal junction (MDJ) region. Of these, $85.1 \%$ entered from lateral and posterior to the ossific nucleus of the capitellum (ONC). The pin angles averaged $58.4^{\circ}$ and $90.5^{\circ}$ in the coronal and sagittal planes respectively. In the second stage, 47 (65.3\%) proximal pins in the intended group exited above the MDJ region, while only 32 (36\%) in the random group exited above the MDJ region.

Conclusion: While aiming at the upper border of the distal MDJ during pinning, lateral pins can easily achieve a higher, proximal exit above the MDJ if inserted from lateral and posterior to the ONC and parallel to the humeral shaft in the sagittal plane. Higher exit can also be easily achieved in younger patients and patients fixated with smaller diameter pins.
\end{abstract}

Keywords: Supracondylar humerus fracture, Pinning fixation, Proximal pin, Entry point, Exit point

* Correspondence: wangenbodor@163.com

Department of Pediatric Orthopedics, Shengjing Hospital of China Medical

University, Shenyang, Liaoning Province, People's Republic of China

(c) The Author(s). 2019 Open Access This article is distributed under the terms of the Creative Commons Attribution 4.0 International License (http://creativecommons.org/licenses/by/4.0/), which permits unrestricted use, distribution, and reproduction in any medium, provided you give appropriate credit to the original author(s) and the source, provide a link to the Creative Commons license, and indicate if changes were made. The Creative Commons Public Domain Dedication waiver (http://creativecommons.org/publicdomain/zero/1.0/) applies to the data made available in this article, unless otherwise stated. 


\section{Introduction}

Supracondylar humeral fracture (SHF) is a common circumstance in the pediatric patients. It accounts for about $60 \%$ of all pediatric elbow fractures [1]. Despite the variety in the severity of fractures and the selection of fixations, Kirschner wire fixation remains the mainstream treatment modality in unstable or displaced SHF. Generally, two or three Kirschner wires inserted from the lateral/medial and lateral condyles and configurated in crossed or divergent manner are said to provide the best stability [2-5].

Lateral entry pinning is however preferred by many surgeons because it can achieve sufficient stability while eliminating the risk of ulnar nerve injury [6-9]. The configuration of the pins should be in accordance with the fracture line to achieve the best stability. Theoretically, the pins should be divergent enough along the fracture line in order to provide the most optimum stability $[10,11]$. However, the entry point at which the proximal lateral pin can be inserted for more proximal exit and therefore attain maximum divergence has not been reported yet in the literature. In some circumstances, like when the fracture lines are more oblique or located in the metaphyseal-diaphyseal junction, this configuration may be difficult to achieve, and therefore, alternative fixation techniques should be sort. In this case, knowing the limits of the exit heights of lateral pins is important for pre-operative planning. In a twodivergent lateral pinning configuration, placement of the proximal lateral pin determines the magnitude of divergence and stability of the fixation. Its limit is therefore of great clinical significance.

This study aimed at analyzing the characteristics of the entry and exit points of the proximal lateral pins and the possible influencing factors, investigating whether the position of the exit points can be intentionally controlled, and finding out how to achieve a higher exit with proximal lateral pins.

\section{Patients and methods}

This study was approved by the Research and Ethics Committee of China Medical University. Data of patients admitted for extension-type SHF between March 2016 and December 2017 were recorded and retrospectively analyzed. The inclusion criteria were as follows: the patients were treated by closed reduction and fixated with two or more Kirschner wires by one of four attending pediatric orthopedic surgeons, who had been specialists for at least 10 years; the Kirschner wires were inserted through a lateral (radial) entry; and the patients had full sets of intraoperative fluoroscopic images in good quality. The exclusion criteria were as follows: the tip of the proximal lateral pin penetrated the anterior/posterior cortex of the humerus on the lateral view X-ray, bent pins lying within the humeri, the images were not in standard anterior-posterior (AP) and lateral views, distal fragment rotation on X-rays, and flexion-type SHF. The surgical procedure and method used to confirm reduction were done as described before [12]. The diameters of the stainless steel K-wires were $1.6 \mathrm{~mm}$ and $2.0 \mathrm{~mm}$ with pyramid tips (Double Engine Medical Material Co., Ltd., Xiamen, China).

In the first stage of the study, data of patients treated between March 2016 and December 2016 were collected and analyzed. All the surgeons were doing the operations freely following the regulations mentioned above and standard treatment protocols. Demographic data relating to age, sex, side of injury, type of fracture, Gartland's classification, location and pattern of fracture line, and pin size used were recorded. The last AP and lateral images before the application of casts were used

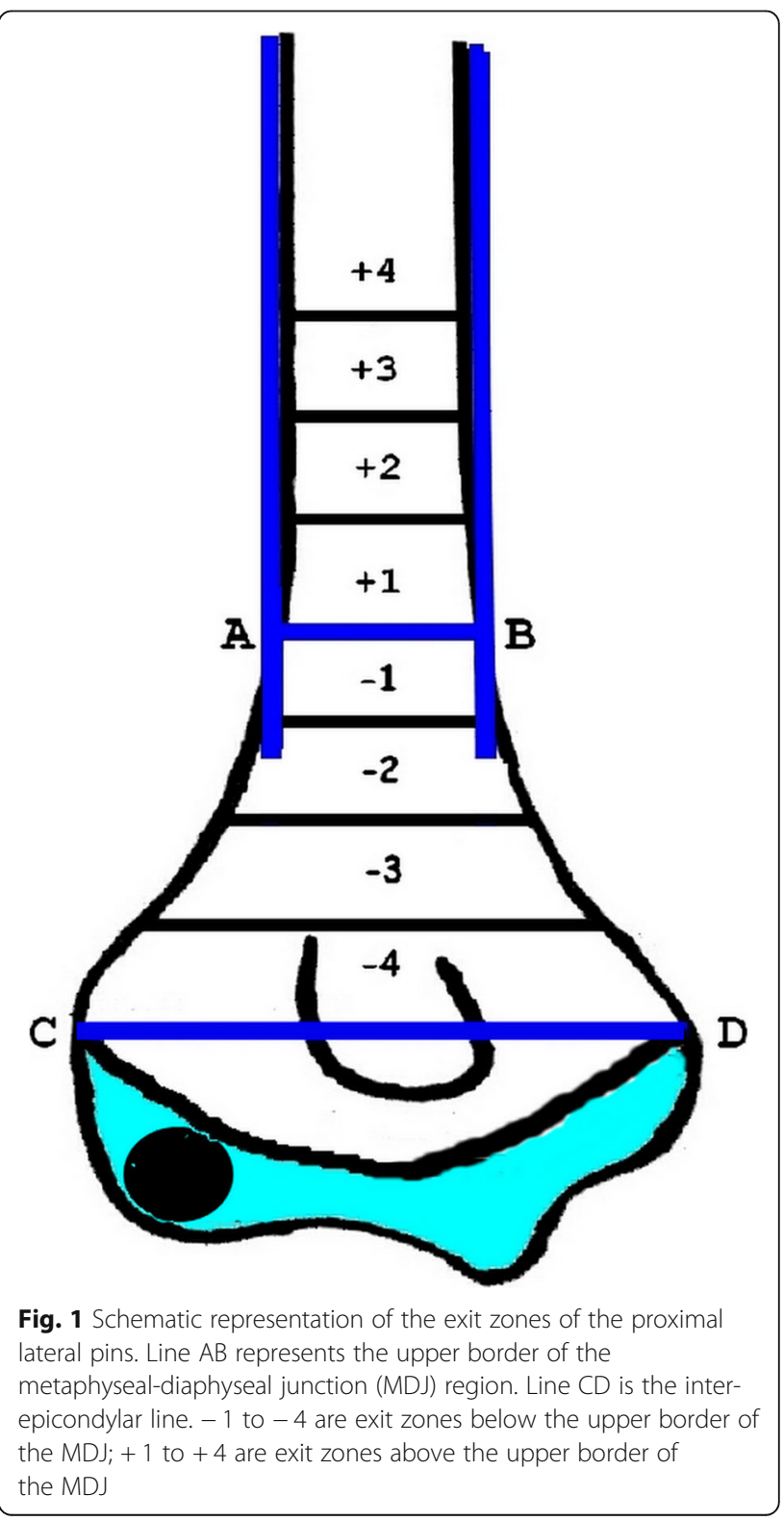




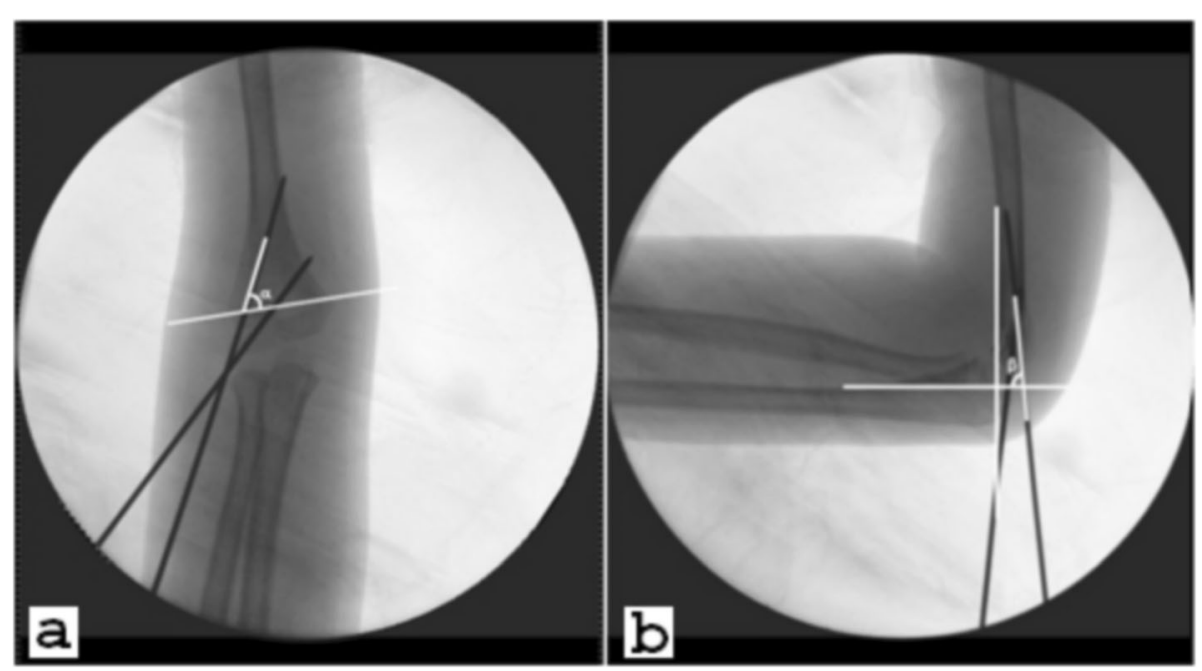

Fig. 2 Pin angles measured on the intra-operative radiographs. a Coronal pin angle $a$ is formed by the proximal pin and a line traversing the maximum diameter of the olecranon fossa (inter-epicondylar line). $\mathbf{b}$ Sagittal pin angle $\beta$ is formed by the proximal pin and a line at the most distal ossified humeral bone edge, which is perpendicular to the anterior humeral line

for observation. Entry points of the proximal lateral pins (the most proximal lateral pin in case of more than two lateral pins) were recorded in reference to the ossific nucleus of the capitellum (ONC) on both views. To determine the exit point of the proximal lateral pin, we first determined and marked the upper border of the distal metaphyseal-diaphyseal junction (MDJ) region by drawing two perpendicular and tangential lines along the shaft of the humerus on the AP radiograph. A horizontal line (line $\mathrm{AB}$ ) passing through the more proximal point of the two points where the two parallel lines intersected the humeral shaft was regarded as the upper border of the MDJ region (Fig. 1). The regions below and above line $\mathrm{AB}$ were further divided into four equal zones $(-4$ to +4 ) as exit zones, based on the distance from the inter-epicondylar line (line $\mathrm{CD}$ ) to the upper border of the $\mathrm{MDJ}$ region (line $\mathrm{AB}$ ) (Fig. 1).

The coronal pin angles $\alpha$, formed by the pin and interepicondylar line (line $C D$ ), were measured on the AP images, while the sagittal pin angles $\beta$, formed by the pin and a line at the most distal ossified humeral bone edge, which was perpendicular to the anterior humeral line, were also measured on the lateral images (Fig. 2). All measurements were done using measurement tools available on the hospital's Picture Archiving and Communication System (PACS) software (Neusoft, Shenyang, China).

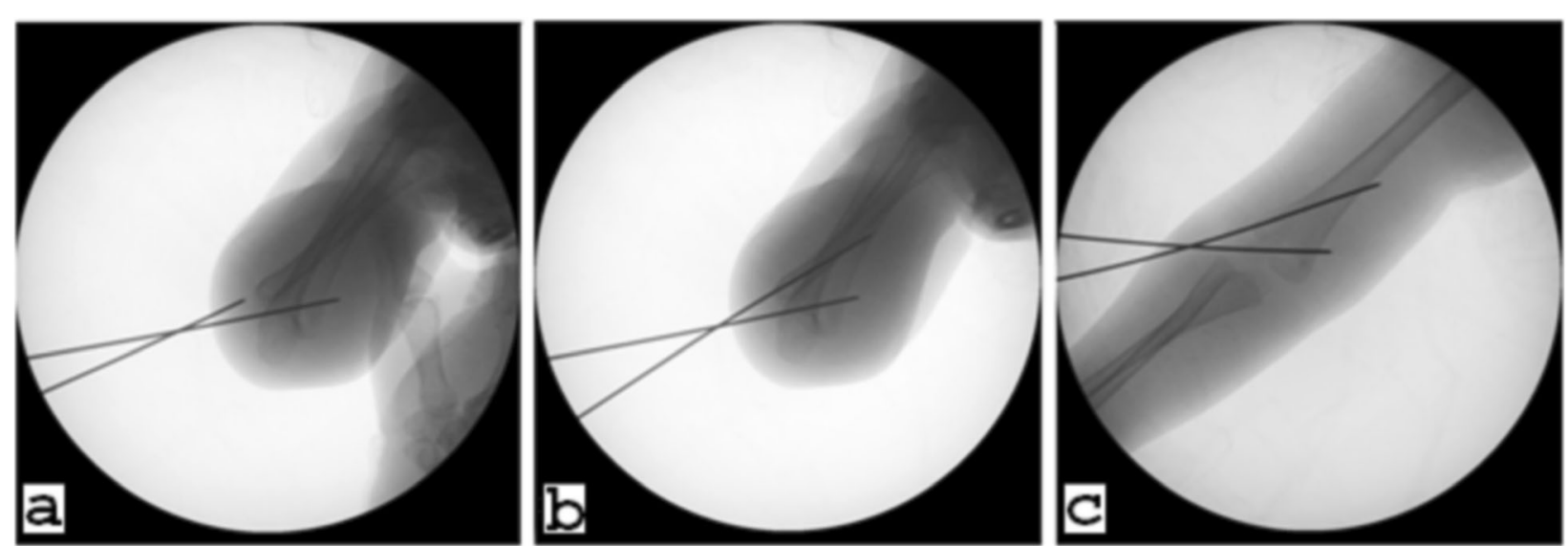

Fig. 3 Placement of the proximal lateral pin was verified under Jones radiograph. a After satisfactorily placing the distal lateral pin, the proximal lateral pin was placed from the lateral distal cortex and aimed to exit in zone +1 in the medial proximal cortex. $\mathbf{b}$ Trajectory of the proximal lateral pin within the distal humerus. c The proximal lateral pin exits the proximal medial cortex of the humerus 
The second stage of this study started from January 2017 and ended in December 2017. Based on the findings of the first stage (see the "Results" section), two of the surgeons (EW and LS), after placing the lower/distal lateral pins, started to insert the proximal lateral pins from lateral (pins laid in the lateral third of the ONC or lateral to the $\mathrm{ONC}$ ) and posterior (pins laid in the posterior third of the $\mathrm{ONC}$ or posterior to the $\mathrm{ONC}$ ) in hyperflexed position under Jones radiographs (Fig. 3), and intentionally aimed at exiting in zone +1 . The location and configuration of the pins were confirmed by intra-operative radiographs. When the lateral pin fixation was found to be satisfactory and stable with no distal fragment rotation, removal and reinsertion of the pins for further proximal exit or insertion of another new pin was avoided. Patients were then immobilized in a long arm cast in 80 to $90^{\circ}$ flexion for a period of 4 to 5 weeks depending on the age of the patient. This group of data was collected as the intended group, while the data of the other surgeons, who continued to fix the fractures according to the regular, standard pinning protocol, were categorized as the random group. Similar demographic data, fracture characteristics, and measurement data were also collected and recorded as in the first stage of the study.

\section{Data analysis}

Statistical analysis of data collected was performed using SPSS version 22 for Windows (IBM Corp., New York, USA). Comparisons of categorical data were performed using the $\chi^{2}$ test and Fisher exact ratio tests. Continuous data were compared through independent-sample $t$ test. Regression analysis was used to determine the associations between pin angles and possible influencing factors. All statistical calculations were performed with a significance level of $p<0.05$.

\section{Results}

Stage I. Characteristics of the proximal lateral pins

Altogether, 161 patients were included in the first stage of the study, with a mean age of 5.2 years (range 1-13 years). Demographic information and the entry and exit points of the pins in both coronal and sagittal planes are listed in Table 1. Eighty-eight (54.7\%) of the pins exited in zone $-1,26(16.1 \%)$ in zone $-2,38(23.6 \%)$ in zone + 1 , and $9(5.6 \%)$ in zone +2 (Fig. 4). The coronal pin angle averaged $58.4^{\circ}$ (range 40.7 to $75.0^{\circ}$ ), while the sagittal pin angle averaged $90.5^{\circ}$ (range 74.3 to $102.0^{\circ}$ ).

We investigated the possible factors that might influence the exit height of the proximal lateral pins. Pin size and age groups made no difference on both the angles and the distribution of pins. In the coronal plane, the medial entry pins made slightly larger coronal angles than that of lateral entry pins $\left(63.9^{\circ} \pm 6.43^{\circ}\right.$ vs. $58.0^{\circ} \pm$ $6.26^{\circ}, p=0.005$ ) (Table 1). In the sagittal plane, the
Table 1 Possible influencing factors of the exit point distribution in the first stage of the study

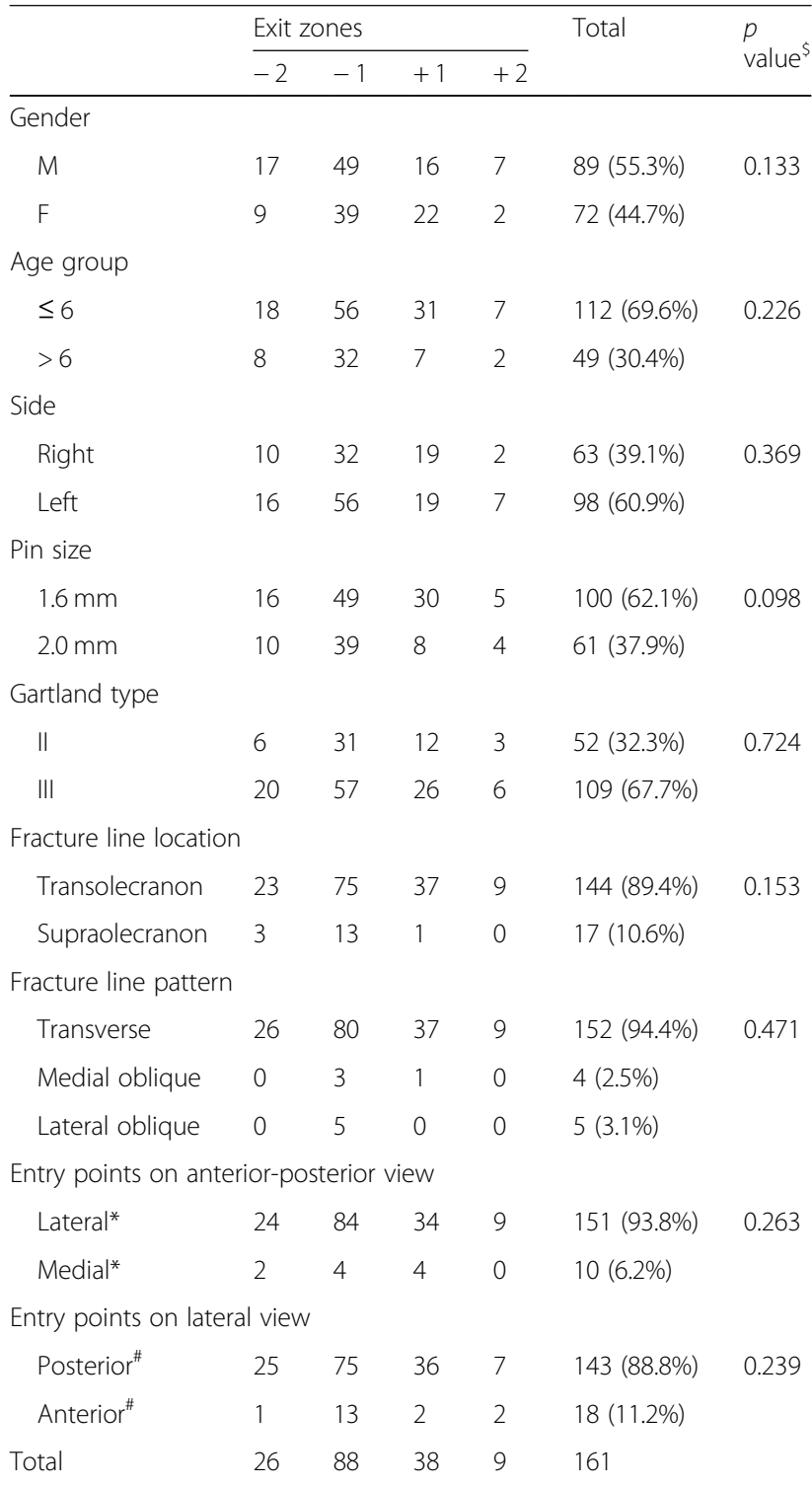

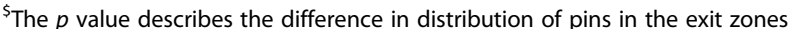
between/among subgroups $\left(x^{2}\right.$ test)

* Lateral: proximal lateral pins laid lateral to the ONC or in the lateral third of the ONC. Medial: proximal lateral pins laid in the medial two thirds of the ONC \#Posterior: proximal lateral pins laid posterior to the ONC or in the posterior third of the ONC. Anterior: proximal lateral pins laid in the anterior two thirds of the ONC

posterior entry pins were tilted more anteriorly than those inside the ONC $\left(89.8^{\circ} \pm 5.96^{\circ}\right.$ vs. $96.2^{\circ} \pm 3.38^{\circ}, p<$ $0.001)$. However, all the abovementioned had no significant difference with regard to the pin distribution in the exit zones (Table 1).

We also tried to find out some characteristic patterns in pins that exited relatively higher. Although the majority of the pins entered from lateral to the $\mathrm{ONC}$ on the $\mathrm{AP}$ view and posterior to it on the lateral view (85.1\%), the statistical difference was insignificant $(p=0.308)$. 


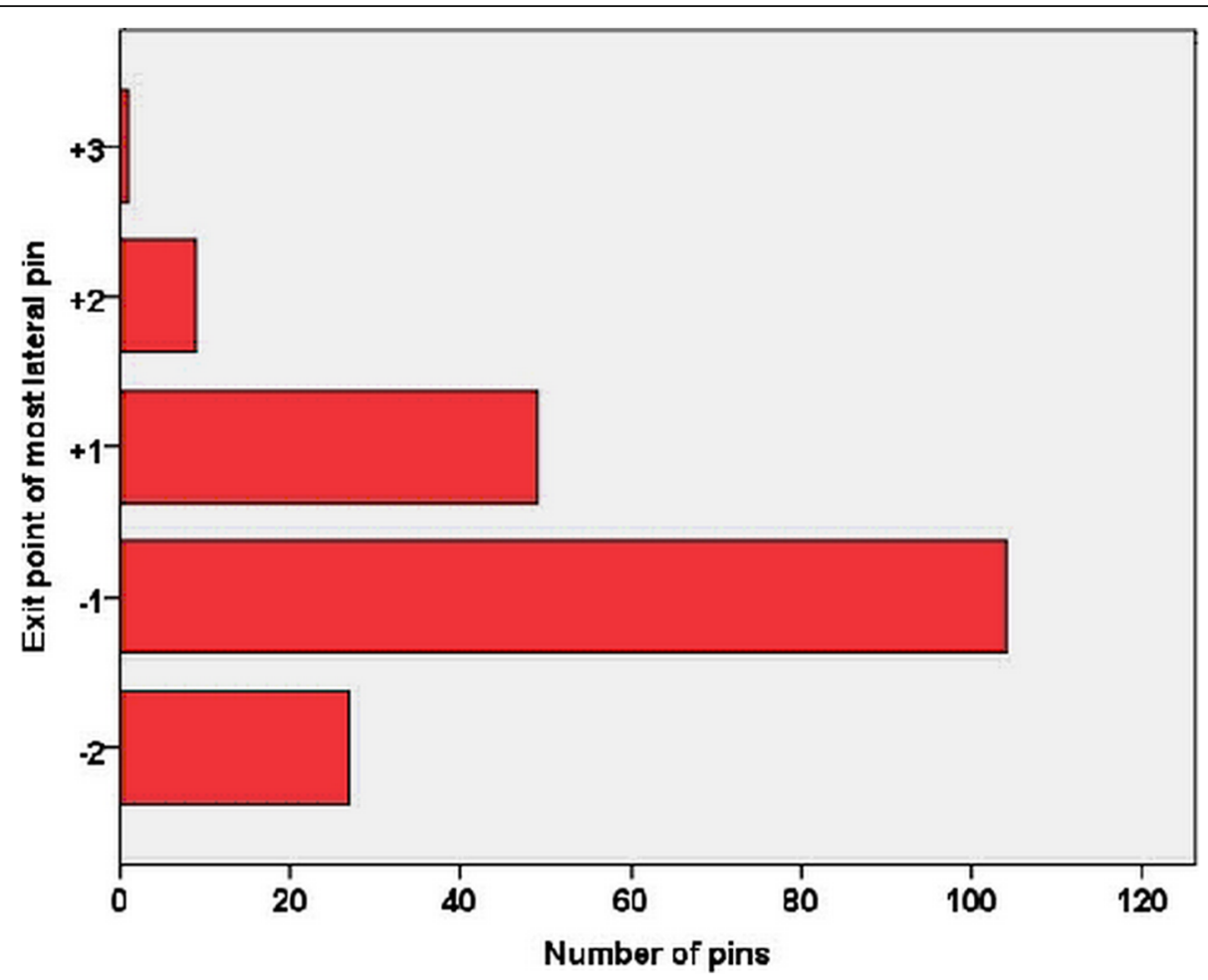

Fig. 4 Distribution of the proximal lateral pins in the exit zones in the first stage of the retrospective study. Only $29.19 \%$ of the pins exited in zone +1 and above

\section{Stage II. Comparison between the random and intended groups}

In the second stage of the study, 161 patients also met our inclusion criteria. All the patients except $3(1.86 \%)$ in the random group who developed a non-significant pin tract infection healed uneventfully and reported no neurovascular complications at the time of pin removal. Demographic information and pin angles for both groups are listed in Table 2. There was no difference in the constitution of the two groups except in pin size and sagittal angle of pins.

In the coronal plane, the angle of the proximal lateral pins averaged $57.9^{\circ}$ (range 34.9 to $70.7^{\circ}$ ) in the random group, compared to $59.7^{\circ}$ (range 46.0 to $71.6^{\circ}$ ) in the intended group. The difference in the comparison of pin angles was statistically insignificant. Distribution of the proximal lateral pins in the exit zones was significantly different between the two groups $(p=0.006)$ (Table 2, Fig. 5). In the sagittal plane, the angle of the proximal pins averaged $90.4^{\circ}$ (range 78.5 to $109.4^{\circ}$ ) in the random group, compared to $88.5^{\circ}$ (range 75.0 to $101.0^{\circ}$ ) in the intended group, which were significantly different $(p=$ 0.031 ). Age group and pin size seemed to have some influence on the exit point distribution of the pins in the intended group, with $p$ values of 0.046 and 0.012 , respectively (Table 2).

\section{Discussion}

The first stage of this study depicted the distribution of the proximal lateral pins used in lateral pinning of supracondylar fracture of the humerus in children. We noticed that $85 \%$ of the proximal lateral pins that exited above the MDJ region were inserted from lateral (pins laid in the lateral third of the $\mathrm{ONC}$ or lateral to the $\mathrm{ONC}$ ) and posterior (pins laid in the posterior third of the $\mathrm{ONC}$ or posterior to the $\mathrm{ONC}$ ). This entry point was therefore adopted for the pinning protocol in the second stage of the study. The second stage of this study demonstrated that surgeons can intentionally obtain higher exit with lateral pins when using this entry point, therefore achieving optimum divergence and greater stability.

Lateral pinning alone is a widely used pinning protocol in the treatment of unstable supracondylar humeral fractures [12-14]. Multiple studies have shown that both crossed and lateral pinning configurations are comparable in terms of stiffness. However, because crossed pins are the most implicated in iatrogenic ulnar nerve injury, some of these studies have therefore advocated for lateral pinning alone as the preferred pinning technique for SHFs, as it offers comparable stability as crossed pins and has reduced or no risk of iatrogenic ulnar nerve injury [3, 6-9]. Loss of reduction is another theoretical complication of pinning fixation method which has been 
Table 2 General information of the random and intended groups in the second stage of the retrospective study

\begin{tabular}{|c|c|c|c|c|}
\hline & Random & Intended & Total & $p$ value \\
\hline Age (years) & $5.1 \pm 3.01$ & $4.8 \pm 2.82$ & $5.0 \pm 2.92$ & 0.532 \\
\hline \multicolumn{5}{|l|}{ Gender } \\
\hline M & 55 & 34 & 89 & \multirow[t]{2}{*}{0.064} \\
\hline $\mathrm{F}$ & 34 & 38 & 72 & \\
\hline \multicolumn{5}{|l|}{ Side } \\
\hline Right & 39 & 27 & 66 & \multirow[t]{2}{*}{0.418} \\
\hline Left & 50 & 45 & 95 & \\
\hline \multicolumn{5}{|l|}{ Gartland type } \\
\hline$\|$ & 20 & 22 & 42 & \multirow[t]{2}{*}{0.245} \\
\hline III & 69 & 50 & 119 & \\
\hline \multicolumn{5}{|l|}{ Fracture line location } \\
\hline Transolecranon & 82 & 66 & 148 & \multirow[t]{2}{*}{0.569} \\
\hline Supraolecranon & 7 & 6 & 13 & \\
\hline \multicolumn{5}{|l|}{ Fracture line pattern } \\
\hline Transverse & 86 & 70 & 156 & \\
\hline Medial oblique & 1 & 1 & 2 & \multirow[t]{2}{*}{0.913} \\
\hline Lateral oblique & 2 & 1 & 3 & \\
\hline \multicolumn{5}{|l|}{ Pin size } \\
\hline $1.6 \mathrm{~mm}$ & 49 & 64 & 113 & \multirow[t]{2}{*}{$<0.001$} \\
\hline $2.0 \mathrm{~mm}$ & 40 & 8 & 48 & \\
\hline Coronal pin angle $a\left(^{\circ}\right)$ & $57.9 \pm 6.70$ & $59.7 \pm 6.29$ & $58.7 \pm 6.57$ & 0.073 \\
\hline Sagittal pin angle $\beta\left(^{\circ}\right)$ & $90.4 \pm 5.88$ & $88.5 \pm 5.59$ & $89.5 \pm 5.82$ & 0.031 \\
\hline
\end{tabular}

$p$ value refers to the comparison between the random and intended groups $\left(x^{2}\right.$ test or independent-sample $t$ test) attributed to many factors [10,11, 15-18]. Though both crossed and lateral pinning configurations have been associated with loss of reduction, the incidence is a little higher with lateral pins alone [7]. For a mechanically sound fixation with lateral pins, two or three pins should be divergently constructed [13]. Choosing the right entry/starting point of the proximal lateral pin is very essential in ensuring maximum divergence and reducing loss of reduction. However, in some cases of high or oblique fracture lines, a proper divergence with bi-cortical purchase of both fragments would be somehow difficult to achieve, or even if achieved may not offer adequate stability to the fracture. In this circumstance, a clear idea about the highest exit point of lateral pins would greatly facilitate pre-operative selection of internal fixation or pin configuration and help reduce reduction loss.

By observing 161 cases fixated with lateral pinning in the first stage, the "natural" distribution of the exit points was depicted. In more than half of the cases, the proximal lateral pins exited from slightly below the MDJ line. A great majority of those that exited above the MDJ line entered from lateral and posterior to the ONC. We therefore hypothesized that the lateral and posterior region in reference to the ONC was an ideal region for inserting lateral pins when higher exit plus maximum divergence is required. The second stage of this study was therefore designed.

According to the second stage of the study, the coronal pin angles were greater in the intended group compared to the random group, indicating that the surgeons were at least partly able to direct the pins toward the intended position despite the variability in bone morphology and other factors such as swelling of the elbow
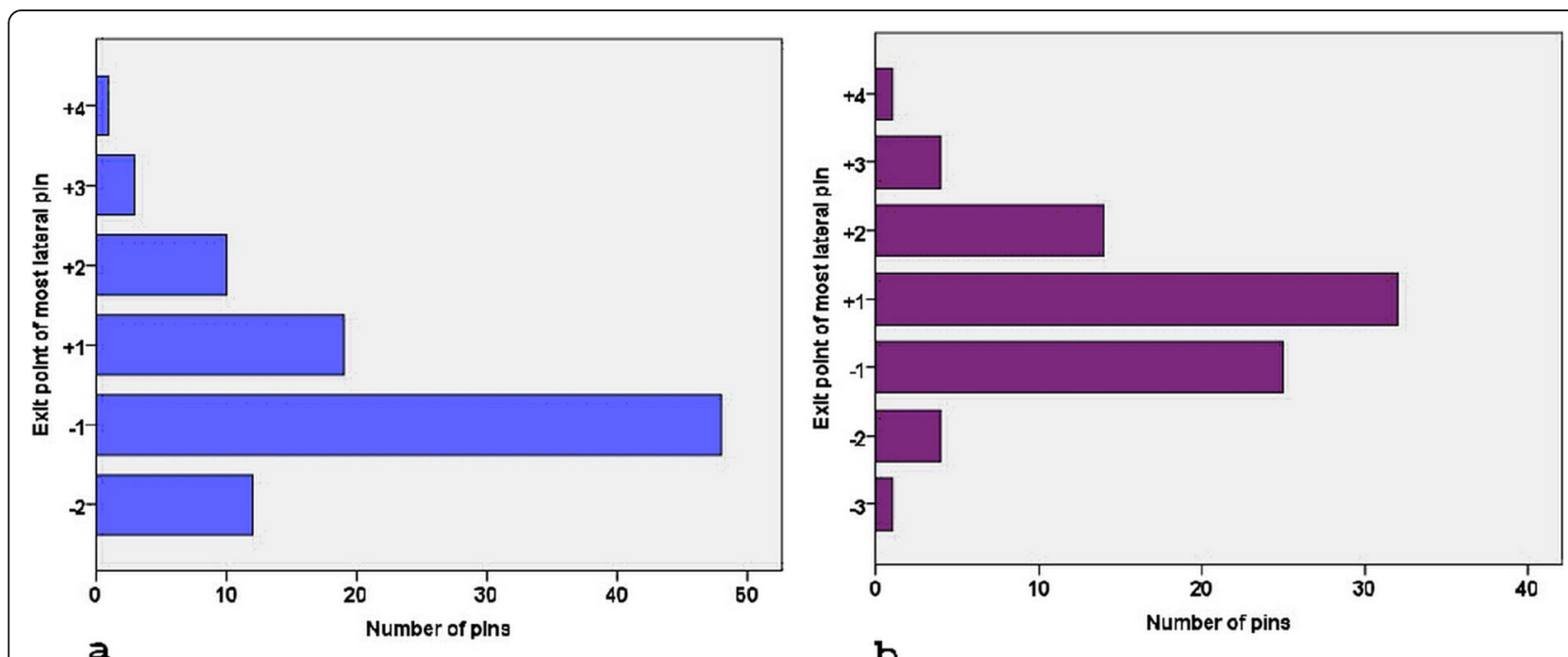

Fig. 5 Distribution of the proximal pins in the second stage of the retrospective study a random group and $\mathbf{b}$ intended group. Distribution of the proximal lateral pins in the exit zones was significantly different between the two groups, $p=0.006$ 
and the effort to limit X-ray exposure. It might sound impractical to focus on angles in clinical practice because it was impossible to precisely pre-determine the pin angles during surgery. So we believe that using the upper borderline of the MDJ as a reference would be more applicable. Forty-seven out of the 72 pins in the intended group exited above the line, compared to 32 out of 89 in the random group. The majority of highly exiting pins in the second stage of the study were seen distributed above and below zone +1 . This demonstrates that aiming at the upper borderline of the MDJ during pin insertion would result in higher exits. In the first stage of the study, we did not find any factors that might influence the height of the exit points. In the intended group of the second stage, however, we found out that the exit points tended to be higher in younger patients and with smaller diameter $(1.6 \mathrm{~mm})$ pins. The bones are more soft and elastic in younger patients than in older patients, which likely made it easier for the pins to penetrate the cortex at a sharp angle. The influence of pin size, however, might also be associated with age because the $2.0-\mathrm{mm}$ pins were generally used in older patients. The $2.0-\mathrm{mm}$ pins were scarcely driven to the region above zone +1 despite the effort to enlarge the coronal pin angles. Whether this was due to the more rigid bones of the patients or the characteristic of the $2.0-\mathrm{mm}$ pins themselves still requires further investigation.

There were however some limitations in this study. The limited sample size in some particular groups made it hard to identify statistical significance of relevant comparisons. Only the proximal lateral pin was evaluated in this study, which we believe is the most important in determining divergence of lateral pins than the distal pin. Moreover, influence of pin numbers and configuration was not included in this study. Randomized controlled prospective studies with larger sample sizes and more standardized operative techniques are warranted to better understand these factors.

\section{Conclusion}

This study analyzed entry points and the distribution of the exit points of proximal pins of lateral pinning alone. With intention, the exit points can be elevated to above the upper border of the MDJ. The ideal entry point to achieve that is if the pin is inserted lateral and posterior to the ONC. Higher exit point can be easily achieved in younger patients and patients fixated with smaller diameter pins. Our findings would be helpful in the preoperative planning and selection of internal fixations in challenging supracondylar humeral fractures.

\section{Abbreviations}

AP: Anterior-posterior; MDJ: Metaphyseal-diaphyseal junction; ONC: Ossific nucleus of the capitellum; SHF: Supracondylar humerus fracture

\section{Acknowledgements}

Not applicable.

\section{Authors' contributions}

All authors made substantive intellectual contributions to this study to qualify as authors. XJ, AK, EW, and TL designed the study. XJ, AK, and TL did acquisition, analysis, and interpretation of data. An initial draft of the manuscript was written by AK. TL re-drafted parts of the manuscript and provided helpful advice on the final revision. All authors were involved in writing the manuscript and read and approved the final version for submission.

\section{Funding}

There was no funding source for this study.

\section{Availability of data and materials}

The datasets in this study are available from the corresponding author on reasonable request.

\section{Ethics approval and consent to participate}

The Medical Ethics Committee of Shengjing Hospital of China Medical University approved this study (reference number 2018PS361K). All procedures performed in this study involving human participants were in accordance with the ethical standards of the institutional review board and ethics committee and with the 1964 Helsinki declaration and its later amendments or comparable ethical standards.

\section{Consent for publication}

Not applicable. For this type of study, formal consent is not required.

\section{Competing interests}

The authors declare that they have no competing interests.

Received: 14 May 2019 Accepted: 4 October 2019

Published online: 09 November 2019

\section{References}

1. Houshian S, Mehdi B, Larsen MS. The epidemiology of elbow fracture in children: analysis of 355 fractures, with special reference to humerus fractures. J Orthop Sci. 2001;6:312-5.

2. Weinberg AM, Castellani C, Arzdorf M, Schneider E, Gasser B, Linke B. Osteosynthesis of supracondylar humerus fractures in children: a biomechanical comparison of four techniques. Clin Biomech (Bristol, Avon). 2007:22(5):502-9.

3. Feng C, Guo Y, Zhu Z, Zhang J, Wang Y. Biomechanical analysis of supracondylar humerus fracture pinning for fractures with coronal lateral obliquity. J Pediatr Orthop. 2012;32(2):196-200. https://doi.org/10.1097/BPO. Ob013e318242a99a.

4. Abdel Karim M, Hosny A, Nasef Abdelatif NM, Hegazy MM, Awadallah WR, Khaled A, Azab MA, ElNahal WA, Mohammady H. Crossed wires versus 2 lateral wires in management of supracondylar fracture of the humerus in children in the hands of junior trainees. J Orthop Trauma. 2016;30(4):e123-8. https://doi.org/10.1097/BOT.0000000000000473.

5. Silva M, Knutsen AR, Kalma JJ, Borkowski SL, Bernthal NM, Spencer HT, Sangiorgio SN, Ebramzadeh E. Biomechanical testing of pin configurations in supracondylar humeral fractures: the effect of medial column comminution. J Orthop Trauma. 2013;27(5):275-80. https://doi.org/10.1097/ BOT.0b013e31826fc05e.

6. Prashant K, Lakhotia D, Bhattacharyya TD, Mahanta AK, Ravoof A. A comparative study of two percutaneous pinning techniques (lateral vs medial-lateral) for Gartland type III pediatric supracondylar fracture of the humerus. J Orthop Traumatol. 2016;17:223-9. https://doi.org/10.1007/ s10195-016-0410-2.

7. Dekker AE, Krijnen P, Schipper IB. Results of crossed versus lateral entry Kwire fixation of displaced pediatric supracondylar humeral fractures: a systematic review and meta-analysis. Injury. 2016;47:2391-8. https://doi.org/ 10.1016/j.injury. 2016.08.022.

8. Begovic N, Paunovic Z, Djuraskovic Z, Lazovic L, Mijovic T, Babic S. Lateral pinning versus others procedures in the treatment of supracondylar humerus fractures in children. Acta Orthop Belg. 2016;82(4):866-71. 
9. Na Y, Bai R, Zhao Z, Han C, Kong L, Ren Y, Liu W. Comparison of lateral entry 357 with crossed entry pinning for pediatric supracondylar humeral fractures: a 358 meta-analysis. J Orthop Surg Res. 2018;13(1):68.359. https:// doi.org/10.1186/s13018-018-0768-3.

10. Pennoc AT, Charles M, Moor M, Bastrom TP, Newton PO. Potential causes of loss of reduction in supracondylar humerus fractures. J Pediatr Orthop. 2014;34(7):691e697. https://doi.org/10.1097/BPO.0000000000000154.

11. Gottschalk HP, Sagoo D, Glaser D, Doan J, Edmonds EW, Schlechter J. Biomechanical analysis of pin placement for pediatric supracondylar humerus fractures: does starting point, pin size, and number matter? J Pediatr Orthop. 2012;32(5):445-51. https://doi.org/10.1097/BPO. Ob013e318257d1cd.

12. Skaggs DL, Cluck MW, Mostofi A, Flynn JM, Kay RM. Lateral-entry pin fixation in the management of supracondylar fractures in children. J Bone Joint Surg Am. 2004;86-A(4):702-7.

13. Lee YH, Lee SK, Kim BS, Chung MS, Baek GH, Gong HS, Lee JK. Three lateral divergent or parallel pin fixations for the treatment of displaced supracondylar humerus fractures in children. J Pediatr Orthop. 2008;28(4): 417-22. https://doi.org/10.1097/BPO.0b013e318173e13d.

14. Scaglione M, Giovannelli D, Fabbri L, Dell'omo D, Goffi A, Guido G. Supracondylar humerus fractures in children treated with closed reduction and percutaneous pinning. Musculoskelet Surg. 2012;96(2):111-6. https:// doi.org/10.1007/s12306-012-0204-5.

15. Sankar WN, Hebela NM, Skaggs DL, Flynn JM. Loss of pin fixation in displaced supracondylar humeral fractures in children: causes and prevention. J Bone Joint Surg Am. 2007:89(4):713-7.

16. Srikumaran U, Tan EW, Erkula G, Leet Al, Ain MC, Sponseller PD. Pin size influences sagittal alignment in percutaneously pinned pediatric supracondylar humerus fractures. J Pediatr Orthop. 2010;30(8):792-8. https:// doi.org/10.1097/BPO.0b013e3181f6d3af.

17. Reisoglu A, Kazimoglu C, Hanay E, Agus H. Is pin configuration the only factor causing loss of reduction in the management of pediatric type III supracondylar fractures? Acta Orthop Traumatol Turc. 2017;51(1):34-8. https://doi.org/10.1016/j.aott.2016.11.003.

18. Aarons CE, Lobts C, Chan DB, Landy DC. Repair of supracondylar humerus fractures in children: does pin spread matter? J Pediatr Orthop B. 2012;21(6): 499 e504.

\section{Publisher's Note}

Springer Nature remains neutral with regard to jurisdictional claims in published maps and institutional affiliations.

Ready to submit your research? Choose BMC and benefit from:

- fast, convenient online submission

- thorough peer review by experienced researchers in your field

- rapid publication on acceptance

- support for research data, including large and complex data types

- gold Open Access which fosters wider collaboration and increased citations

- maximum visibility for your research: over $100 \mathrm{M}$ website views per year

At BMC, research is always in progress.

Learn more biomedcentral.com/submissions 